\title{
A Method of Path Planning and Control Strategy for Carrier-Based UAV in Return Section
}

\author{
Chaoying Pei, Jingjuan Zhang, Xueyun Wang ${ }^{*}$ and Qian Zhang \\ School of Instrumentation Science and Opto-electronics Engineering, Beihang University, Beijing 100191, China \\ Corresponding author: Xueyun Wang \\ ${ }^{*}$ Corresponding author
}

\begin{abstract}
Compared with conventional path planning method, the path planning for carrier based UAVs has its own characteristics, especially in its return section. Firstly, the fuel carried with the UAV is limited, so that the UAV is expected to return to the carrier as soon as possible after the task is finished. Secondly, the carrier, which is moving at a certain speed, constrains that the UAV's velocity must be in the same direction with that of the carrier before landing. Thirdly, due to the limited landing space on the carrier, the lateral error between the UAV and the carrier should be minimized. Considering all above, this paper proposes a path planning method aiming at the carrierbased UAV in its return section, and to ensure the UAV to fly along the planned path accurately and with stable attitude, control strategies are designed. To a void unnecessary detours, the velocity of both the UAV and the carrier is taken into consideration and the estimated landing window is updated when either of the velocity changes. The simulation results show the path and control strategy proposed in the paper are effective.
\end{abstract}

Keywords- carrier-based UAV; path planning; accompanying flight; fuzzy controller

\section{INTRODUCTION}

Unmanned aerial vehicles (UAV) are increasingly being used in civil and military applications for dull, dirty and dangerous missions. Modern UAV applications include a wide variety of intelligence missions, such as search and rescue operations, area mapping, agricultural operation [1,2], and marine measurement [3] etc. And in recent years, considerable attention has been given to UAVs capable of performing critical needs for the Navy. These UAVs are capable of takeoff and landing on an aircraft carrier, while the smaller ones are even able to equip the warships [4]. One of the important problems related to naval Unmanned Aerial Vehicles (UAV), is the design of an automatic landing system that would enable autonomous landing of a UAV on an aircraft, and a lot of studies has been done on this problems[5,6]. An innovative autonomous carrier landing system for UAVs is proposed in [5], referred to as the Carrier Motion Prediction \& Autonomous Landing (CM-PAL) system. The system is based on real-time estimation of magnitudes and frequencies of waves encountered by the carrier, and online prediction of the carrier motion. In [6], a linear Model Predictive Control (MPC) design for the helicopter is investigated using linearized models and it is evaluated using the nonlinear model. However, these landing approaches can only be used in the situation where the UAV is close enough to the carrier and the velocity should be in the same direction of the carrier. After the UAV finishes its mission, a path should be planned to guide the UAV to approach the carrier in an energy-saving and safe way, before preparing to land. A serious of researches related to the path planning for UAV have been conducted, but the majority of them are aiming at the obstacle avoidance $[7,8,9]$. Actually, for a carrier-based UAV, which will land on a moving carrier, path planning is not exactly the same. A carrier-based UAV is expected to achieve accompanying flight before landing, which means the velocity of the UAV and the carrier must be in the same direction, and the lateral error between the UAV and the carrier should be minimized so that the UAV can prepare to land. Considering all the aspects above, a method of path planning and control strategy is proposed in the paper.

In the control strategy, the velocity of both UAV and carrier is considered and the meeting point of them is predicted. After the meeting point being estimated, a path is planned for the UAV to get in to the landing window which is always 1 kilometers behind the carrier. Then a fuzzy controller is used to eliminate the lateral error between the carrier and the UAV in the last kilometer, so that the UAV can be prepared for the landing or net-catching.

The remaining part of the paper is organized as follows: In section 2 the definition of coordinate systems, path planning and control strategy framework is presented. Section 3 introduces control strategy and then the guidance algorithms is proposed to generate the command for the actuator of UAV. Numerical simulation, experimental results are performed to verify the performances of the proposed methodology in Section 4. Finally, conclusion and future studies are provided in Section 5.

\section{OVERVIEW OF THE CARRIER-BASED UAV SYSTEM}

\section{A. Configuration of UAV and Coordinate Definition}

To explain the landing approach strategy in this paper clearly, the frames used in this paper are defined as follows. 


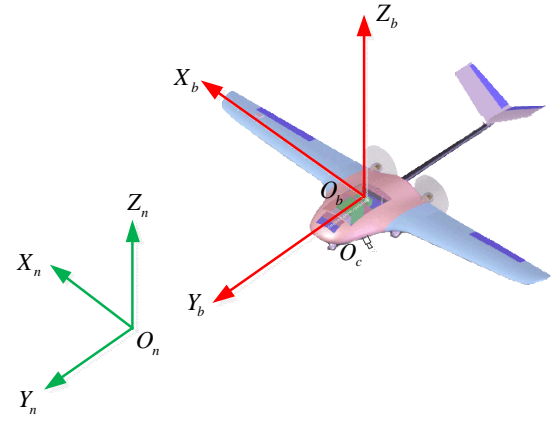

FIGURE I. THE STRUCTURE OF UAV

As is shown in Fig.1, the local geographical coordinate frame is called navigation frame ( $\mathrm{N}$-frame), the $X_{n}$-axis points to east in the local level, $Y_{n}$-axis points to north in the local horizontal and $Z_{n}$-axis is defined according to the righthand rule. The body coordinate frame (B-frame) is fixed to the $\mathrm{UAV}, X_{b}$-axis points to the right of UAV, $Y_{b}$-axis points to the front and $Z_{b}$-axis is defined according to the right-hand rule.

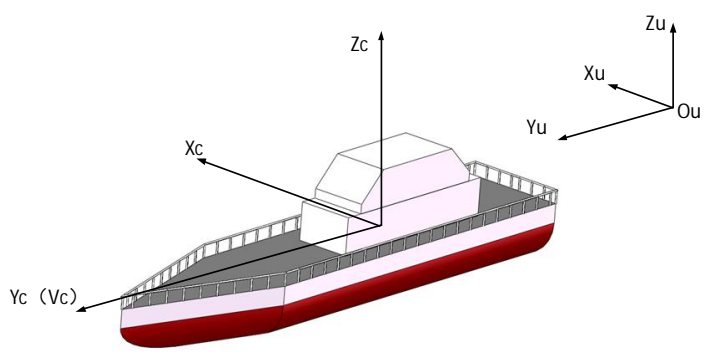

FIGURE II. THE STRUCTURE OF THE CARRIER

At the last stage, UAV reaches the same velocity with carrier, which means implementing accompanying flight and prepares to land. Therefore, the carrier coordinate(C-Frame) is denoted as follows: $Y_{c}$-axis is defined according to the velocity direction of the carrier $V_{c}, Z_{c}$-axis is aligned with $Z_{n}$-axis and $X_{c}$-axis is defined according to the right-hand rule. UFrame is the frame in which the landing window position is estimated, the three axes of U-Frame are aligned with those of C-Frame, but the origin is taken as the position of the UAV.

\section{B. Path Description}

When the UAV has finished the missions, it is expected to return to the carrier safely and quickly, here are several requirements for this stage of flight. 1) Considering the limited energy-carrying capacity of a UAV, the path should ensure the UAV return with the least energy and time consumption. 2) To get into the landing window precisely, the lateral deviation between the UAV and the carrier should be in an acceptable range so that it can further adjust to prepare for the landing. 3) When the UAV gets into the landing window, the velocity of the UAV should be in the same direction with $Y_{c}$-axis, which is also the direction of carrier velocity. To meet the requirements above, we propose a path planning method illustrated in the Fig.3

As is shown in the Fig.3, the landing window is always 1 kilometer behind the carrier, and the UAV gets into the landing window along a circular trajectory, whose radius is selected according to the safe flight attitude of a UAV. Before the UAV start circling, it will approach the landing window in the direction of the circle's tangent. This path is able to guide the UAV to fly with small heading angle increment in each control circle. Consequently, the path can avoid large-maneuver for the UAV, which would also contribute to the energy saving as well as flight security.

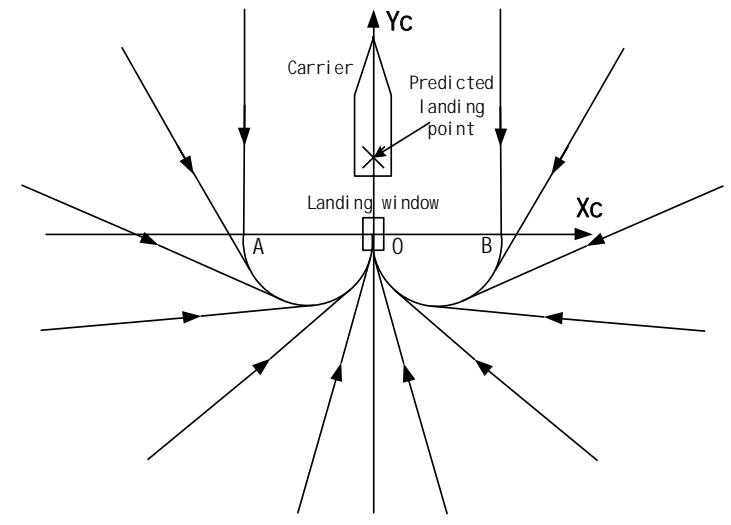

FIGURE III. PATH DESCRIPTION

\section{Path Description}

The flowchart of the proposed framework is shown in Fig.4. The system architecture can be described in brief as landing window estimation, path planning and guidance towards landing window, further lateral errors elimination after getting into the landing window in preparation for landing approach.

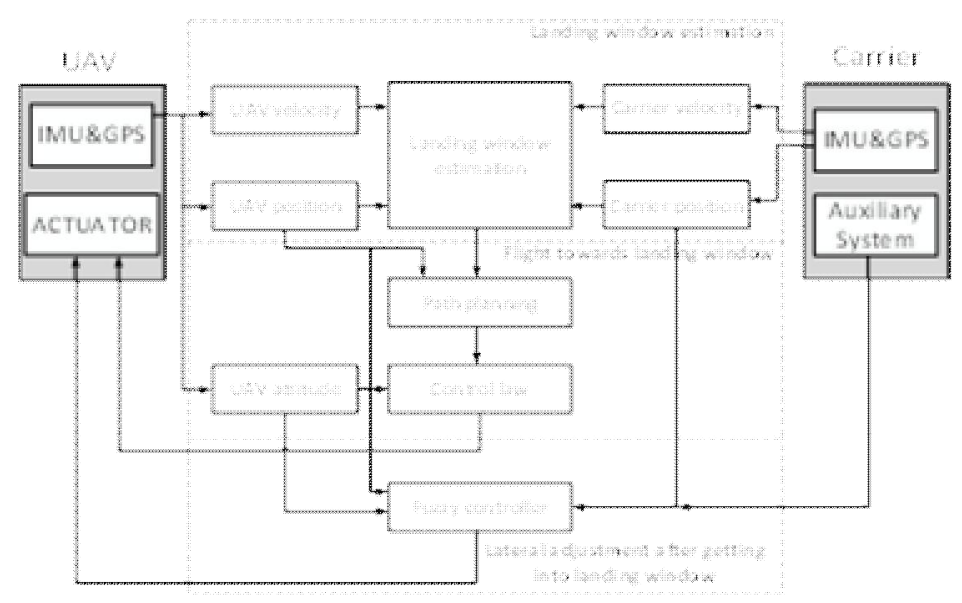

FIGURE IV. FLOWCHART OF THE CONTROL STRATEGY

Process of the UAV landing approach is divided into three steps: 1) According to the current velocity and position of UAV and carrier, the landing window, which is the destination of the 
UAV, is estimated. When the velocity of UAV or carrier changes, the estimated landing window will be updated. 2) After the landing window being estimated, the commands of the UAV are generated based on it, and the UAV is guided to fly along the path which is planned to ensure the UAV get in to the landing window smoothly and with the least energy consumption. And this stage ended with the UAV getting in to the landing window with acceptable deviation and its velocity reaching the same direction as the velocity of carrier. 3) When the UAV gets into the landing window, the lateral errors should be further eliminated by fuzzy controller to prepare for the next landing or net-catching task.

\section{CONTROL SYSTEM DEVElopment}

\section{A. Landing Window Estimation}

Landing window estimation is conducted according to the current velocity and position of both the UAV and the carrier which are provided by their respective GPS and IMU, as is shown in the Fig.5

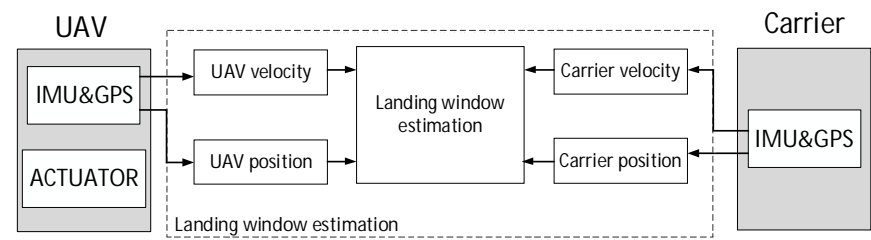

FIGURE V. FLOWCHART OF THE LANDING WINDOW ESTIMATION

The landing window is estimated by the geometric constraints which is illustrated in the Fig.6. Point B indicates the current position of the UAV and point $\mathrm{O}$ indicates the estimated landing window. Point $O_{1}$ indicates the center of the circular trajectory. $\mathrm{R}$ is the circle radius set according to the flight performance of the UAV. If the UAV and the carrier keep moving in the current velocity, the UAV and the landing window will reach point $\mathrm{O}$ together.

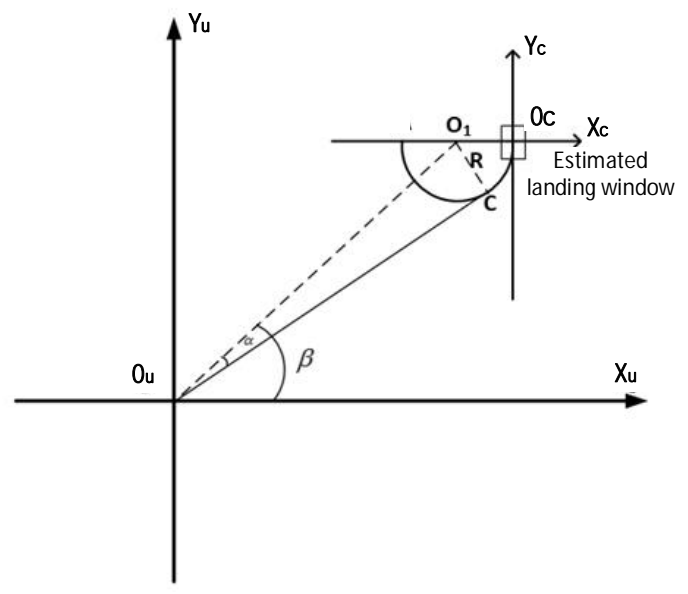

FIGURE VI. ESTIMATION METHOD
However, if the velocity of either UAV or carrier changes, the landing window position will be estimated again to ensure the UAV to fly towards the right destination and through the shortest path. The geometry constraints are conveyed by the following equations:

$$
\begin{array}{r}
x_{t}=x_{0} \\
y_{t}=y_{0}+v_{y} \cdot t
\end{array}
$$

In the equation 3-1 and 3-2, $\left(x_{0}, y_{0}\right)$ is the current position of the landing window which is provided by the GPS\&IMU of the carrier together with the velocity $v_{y},\left(x_{t}, y_{t}\right)$ is the estimated landing window position where the UAV will meet the landing window if the velocity of both the UAV and the carrier do not change. is the duration that is need for the UAV and the landing window to get to the estimated position Oc.

$$
\begin{gathered}
O_{1} O_{u}=\sqrt{x_{t}^{2}+y_{t}^{2}} \\
O_{u} C=\sqrt{O_{1} O_{u}{ }^{2}-R^{2}} \\
\alpha=\arcsin \left(\frac{R}{O_{1} O_{u}}\right) \\
\beta=\arctan \left(\frac{y_{t}}{x_{t}}\right) \\
C O_{c}=R\left(\frac{\pi}{2}-\beta+\alpha\right) \\
O_{u} C+C O_{c}=V a \cdot t
\end{gathered}
$$

Equations above illustrate the geometrical relations should be fitted. In these equations, $t$ is the only unknown, and can be solved by simultaneous equation. After solving $t$, the estimated position of the landing window can be easily calculated.

\section{B. Guidance and Control Law}

In this section, the guiding method for UAV is described. Method in this paper is aimed at fixed-wing aircraft, and the three attitude channels of the UAV are decoupled and could be 
controlled separately. The process of the guidance can be divided into 3 stages, as is shown in the Fig.7: 1) Guidance before the UAV start circle and fly towards the direction of the tangent of the circular trajectory. 2) Guidance during the hover which is the transition stage to the landing window. 3) Guidance after the UAV get into the landing window and in this stage the main challenge for the controller is to eliminate lateral errors.

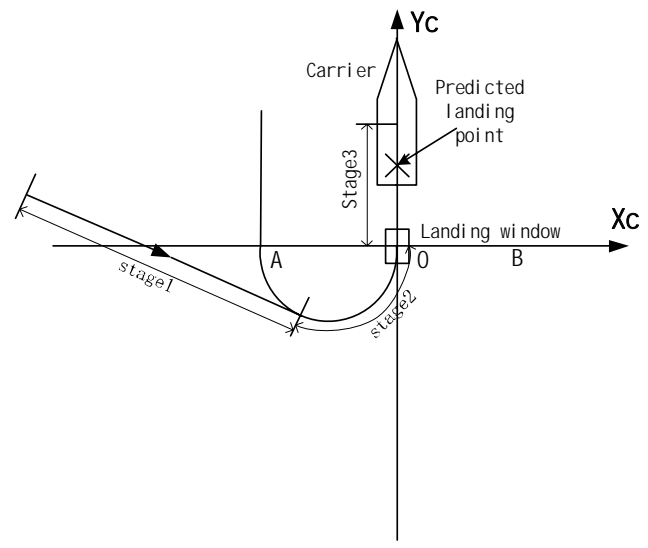

FIGURE VII. THREE STAGES IN THE PROCESS OF APPROACH

To complete the trajectory tracking and eliminate the lateral error at the last stage, the control law is designed as follows: To avoid unnecessary climb and keep the stability of the UAV, the altitude and pitch angle are expected to keep invariable. Here, a PID controller is used to control the elevator of the UAV with deviations of pitch angle and altitude as inputs and the deflection angle of the elevator as the output. Fig. 8 indicates the pitch channel control method with three feedback loops.

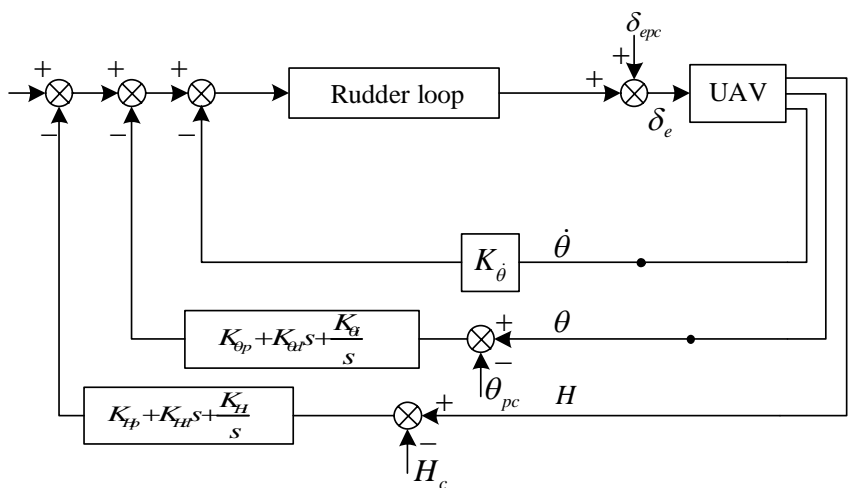

FIGURE VIII. FLOWCHART OF THE PITCH CHANNEL CONTROLLER

To keep the UAV with a fixed and stable pitch angle, the speed should also be invariable. Another PID controller is designed to realize it, as is shown in the Fig.9. The velocity command is computed according to the trim for lift and gravity.

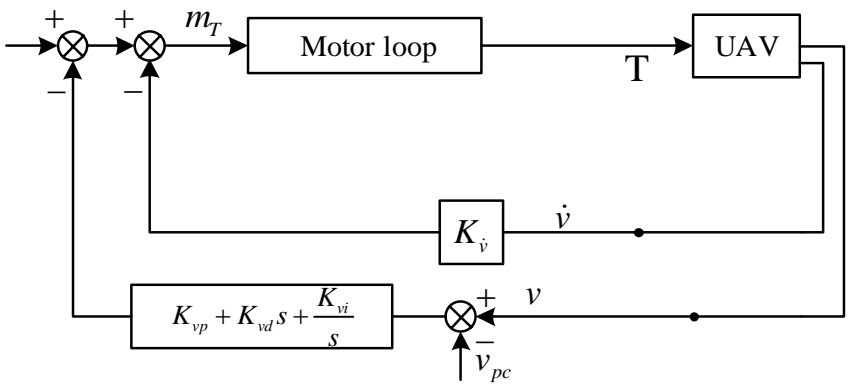

FIGURE IX. FLOWCHART OF THE VELOCITY CHANNEL CONTROLLER

- Control law for the first stage

In the first stage, the UAV approach the estimated landing window before it starts circling, as is shown in the Fig.7. The heading command, which is pointing to the tangent of the circle in stage 3 , can be calculated by the following method, as is shown in the Fig.10.

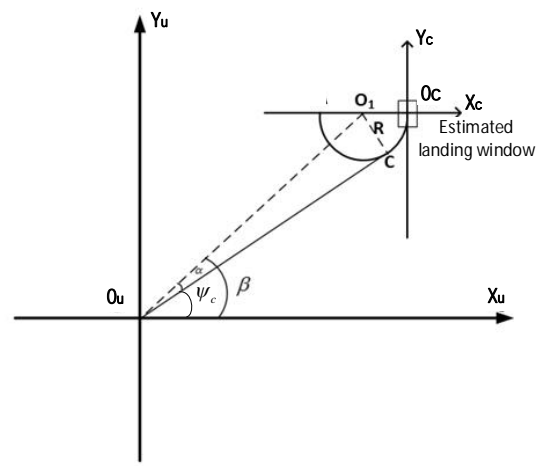

FIGURE X. METHOD OF CALCULATION OF HEADING COMMAND

$$
\begin{gathered}
\beta=\arctan \left(\frac{y_{O_{1}}-y_{O_{u}}}{x_{O_{1}}-x_{O_{u}}}\right) \\
\alpha=\arcsin \left(\frac{R}{O_{1} O_{u}}\right)
\end{gathered}
$$

$$
\psi_{c}=\arctan \left(\frac{y_{O_{1}}-y_{O_{u}}}{x_{O_{1}}-x_{O_{u}}}\right)-\arcsin \left(\frac{R}{O_{1} O_{u}}\right)
$$

When the UAV is flying along a curve trajectory, the roll angle, which can provide the UAV with centripetal force, should response to the turning. The relationship of the roll angel and the curvature is shown in the Fig. 11. 


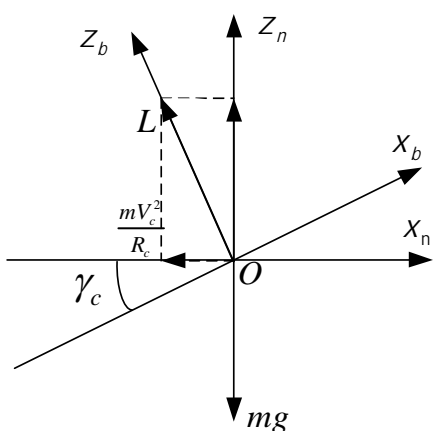

FIGURE XI. UAV FORCE DIAGRAM

In the Fig.11, $m g$ is the gravity, $\gamma_{c}$ is the roll angle, $R_{c}$ is the turning radius, and $\mathrm{L}$ is the aerodynamic lift which will balance the gravity as well as provide the centripetal for the UAV's turning motion. The command of roll angle can be calculated by the following formula:

$$
\gamma_{C}=a \tan \left(\frac{V_{c}^{2}}{R \times g}\right)
$$

In this formula, $V_{c}$ is the horizontal velocity which can be obtained by the airborne INS, however, the turn radius, $\mathrm{R}$, is difficult to calculate in real-time. To calculate the command of roll angle, the formula can be transformed to be as follow:

$$
\gamma_{C}=a \tan \left(\frac{V_{c} \cdot \omega}{g}\right)
$$

In this formula, $\omega$ is the yaw angle velocity which can also be obtained by the airborne INS. But considering the possible wobble of $\omega$, which would also cause the wobble of the roll angle and lead to the attitude instability, $\omega$ is expressed as:

$$
\omega=\frac{\psi_{c(i)}-\psi_{c(i-1)}}{d t}
$$

Roll command can be calculated by:

$$
\left.\gamma_{C}=a \tan \left[\frac{V_{c} \cdot\left(\psi_{c(i)}-\psi_{c(i-1)}\right)}{g \cdot d t}\right)\right]
$$

Here, $d t$ is the control circle of the system, $\psi_{c(i)}$ and $\psi_{c(i-1)}$ is the command of heading angle in two adjacent control circles. Calculating the roll angle from the command of heading angle can not only avoid the Gyro noise causing wobble in the roll channel, but also, by adjusting the roll angle according to the expected heading angle, the control system can guide the UAV to turn more precisely as needed. The flowchart of the control law for the first stage can be expressed as follow:

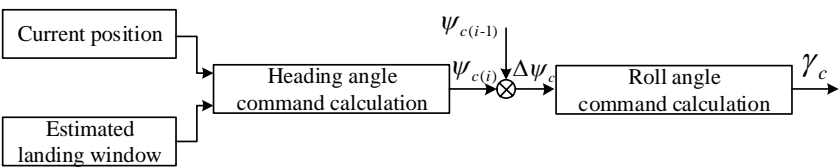

FIGURE XII. CONTROL LAW FOR THE FIRST STAGE

The roll channel and the heading channel are controlled with controllers with feedback, as is shown in the Fig.13 and Fig.14.

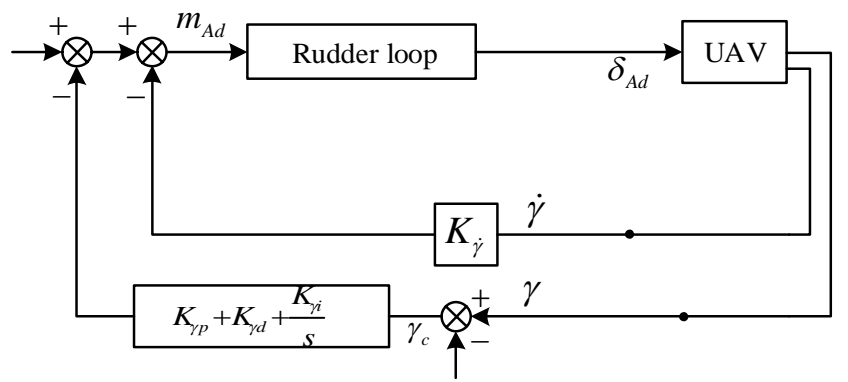

FIGURE XIII. CONTROLLER FOR THE ROLL CHANNEL

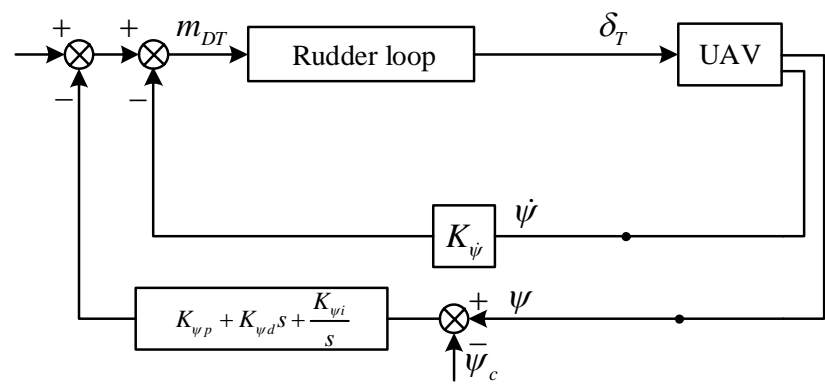

FIGURE XIV. CONTROLLER FOR THE HEADING CHANNEL

- Control law for the second stage

In the second stage, the UAV starts circling and at the end of this stage, it will get into the landing window with acceptable lateral deviation and the velocity of the UAV is essentially in the same direction with that of the carrier.

To ensure the UAV circle in the pre-selected orbit, the process of the second stage are divided into three cases according to the radius of actual circle radius R. Fig. 15 . illustrates the three cases of this stage, in which $R 1, R 2, R 3$ is the radius of the actual route in three cases respectively, $R_{c}$ is the radius of planned route. 


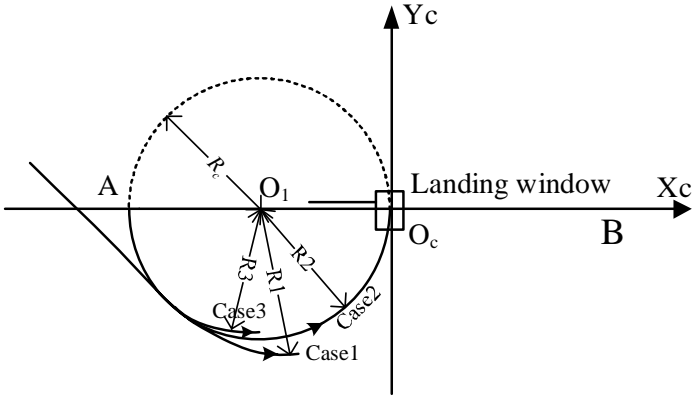

FIGURE XV. THREE CASES IN THE SECOND STAGE

1)Case1: $R>R_{c}$

In the case1, distance between the UAV and the center is further than $R_{c}$, which means that the UAV flies out of the planned circular route. In the circumstances, the control approach is the same as that of the first stage, in which the UAV fly in tangent direction of the planned circular trajectory.

2)Case2: $R_{c}-\Delta R<R<R_{c}$

In the case 2 , distance between the UAV and the center is shorter than $R_{c}$, but the error is small and acceptable. In the circumstances, the UAV is expected to circle at a fixed angle velocity, therefore the roll angle is also fixed. The heading angle command can be calculated by following formula:

$$
\psi_{c(i)}=\psi_{c(i-1)}+\omega \cdot \mathrm{Dt}
$$

3)Case3: $R<R_{c}-\Delta R$

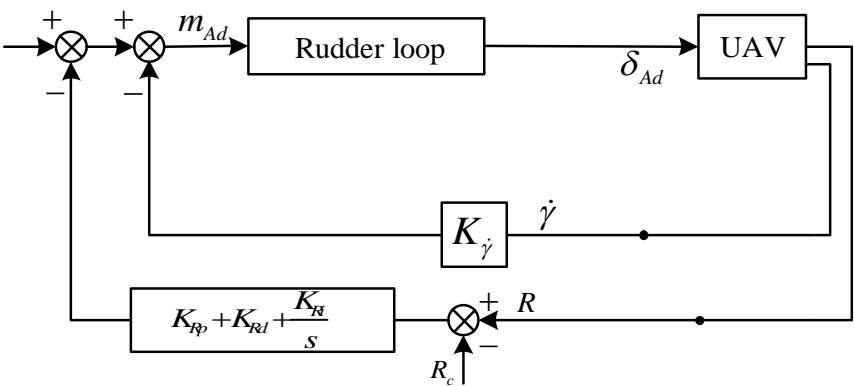

FIGURE XVI. CONTROLLER OF THE RADIUS CHANNEL

In case 3, the roll angle is controlled following the flowchart above. In this case, the UAV flies too close to the center. To get the UAV back to the planned trajectory, the roll angle decreases so that the centripetal force will also decrease which will cause the increase of the radius of circle motion and the flight situation will get back to the case1 or case2.

\section{Further Elimination of the Lateral Error}

After getting into the landing window, the UAV starts preparing for its final approach which will complete with the assistance of Radar vectoring, Photoelectric guidance, etc. Before the final approach, the lateral errors should be eliminated as much as possible. Considering the nonlinear relationship between the control mechanism and the lateral error, here we use a fuzzy controller to improve the lateral accuracy. The flowchart is shown as follow:

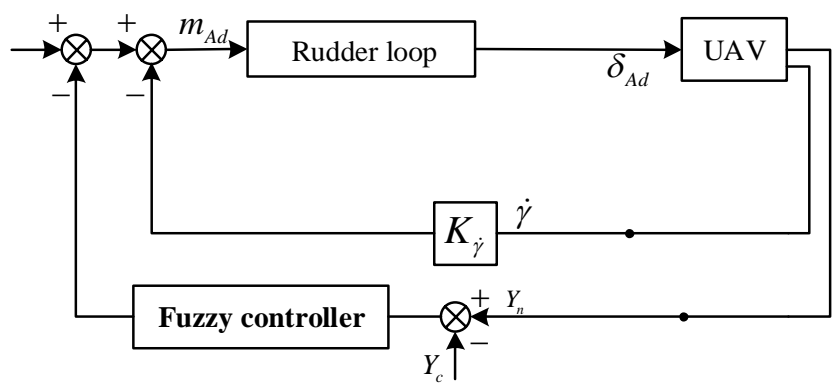

FIGURE XVII. FUZZY CONTROLLER FOR LATERAL ERROR ELIMINATION

$Y_{n}$ is the actual lateral position of the UAV while $Y_{c}$ is the expected lateral position. By increasing the roll angle according to the lateral error, the component of the lift will provide the UAV with lateral acceleration and therefore eliminate the error.

\section{SimUlATION RESUlT}

In three stages of the guidance, the pitch angle and the velocity are controlled in the same way, which can be seen in the Fig.8. and Fig.9. In simulation, the parameters of the PID controllers are designed as follows:

TABLE I. PID PARAMETERS OF PITCH CHANNEL

\begin{tabular}{|c|c|c|c|}
\hline Pitch angle command & $K_{\theta p}$ & $K_{\theta d}$ & $K_{\theta i}$ \\
\hline $2^{\circ}$ & 3 & 0.2 & 2 \\
\hline
\end{tabular}

TABLE II. PID PARAMETERS OF HEIGHT CHANNEL

\begin{tabular}{|c|c|c|c|}
\hline Height command & $K_{H p}$ & $K_{H d}$ & $K_{H i}$ \\
\hline 200 & 0.05 & 0 & 0.05 \\
\hline
\end{tabular}

TABLE III. PID PARAMETERS OF VELOCITY CHANNEL

\begin{tabular}{|c|c|c|c|}
\hline Trim velocity & $K_{v p}$ & $K_{v d}$ & $K_{v i}$ \\
\hline $37.46 \mathrm{~m} / \mathrm{s}$ & 0.1 & 0.001 & 0.2 \\
\hline
\end{tabular}

\section{A. Parameters of the Controllers for the First Stage}

In the first stage, two PID controllers are designed respectively for roll channel and heading channel to ensure the UAV to approach the carrier smoothly. The roll angle command and the heading angle command are calculated by the method above. And the parameters of the controllers are set as follows: 
TABLE IV. PID PARAMETERS OF ROLL CHANNEL

\begin{tabular}{|c|c|c|c|}
\hline$K_{\gamma p}$ & $K_{\gamma d}$ & $K_{\gamma i}$ & $K_{\gamma p}$ \\
\hline 3.5 & 1.6 & 1.5 & 3.5 \\
\hline
\end{tabular}

TABLE V. PID PARAMETERS OF HEADING CHANNEL

\begin{tabular}{|c|c|c|}
\hline$K_{\psi p}$ & $K_{\psi d}$ & $K_{\psi i}$ \\
\hline 42 & 5 & 0 \\
\hline
\end{tabular}

B. Parameters of the Controllers for the Second Stage

In the second stage, there are three cases using different control strategies which can keep the UAV fly along the circular trajectory. The three cases can be seen in the Fig.15.

- Controllers for case 1

Case1: $R>R_{c}$. The controller is the same as that of the first stage:

TABLE VI. PID PARAMETERS OF ROLL CHANNEL

\begin{tabular}{|c|c|c|}
\hline$K_{\gamma p}$ & $K_{\gamma d}$ & $K_{\gamma i}$ \\
\hline 3.5 & 1.6 & 1.5 \\
\hline
\end{tabular}

TABLE VII. PID PARAMETERS OF HEADING CHANNEL

\begin{tabular}{|c|c|c|}
\hline$K_{\psi p}$ & $K_{\psi d}$ & $K_{\psi i}$ \\
\hline 42 & 8 & 0 \\
\hline
\end{tabular}

- Controllers for case2

Case2: $R_{c}-\Delta R<R<R_{c}$. The roll angle command is calculated according to the horizontal velocity of the UAV and the expected turn radius, which is fixed in this this case. The heading angle command is accumulated with fixed angle according to the expected circle angular velocity. The parameters of the controller are set as follows:

TABLE VIII. PID PARAMETERS OF ROLL CHANNEL

\begin{tabular}{|c|c|c|c|}
\hline Roll angle command & $K_{\gamma p}$ & $K_{\gamma d}$ & $K_{\gamma i}$ \\
\hline$-15.99^{\circ}$ & 3.5 & 1.6 & 1.5 \\
\hline
\end{tabular}

TABLE IX. PID PARAMETERS OF VELOCITY CHANNEL

\begin{tabular}{|c|c|c|c|}
\hline Circle angular velocity & $K_{\psi p}$ & $K_{\psi d}$ & $K_{\psi i}$ \\
\hline $4.29^{\circ} / \mathrm{s}$ & 42 & 8 & 0 \\
\hline
\end{tabular}

- Controllers for case3

Case3: $R<R_{c}-\Delta R$. In case3, the input of the PID controller is the error of the turn radius and the output is the deviation of the ailerons.

TABLE X. PID PARAMETERS OF VELOCITY CHANNEL

\begin{tabular}{|c|c|c|c|}
\hline Expected radius & $K_{\psi p}$ & $K_{\psi d}$ & $K_{\psi i}$ \\
\hline $500 \mathrm{~m}$ & 0.04 & 0.002 & 0.0001 \\
\hline
\end{tabular}

\section{Parameters of the Fuzzy Controller for the Third Stage}

In the third stage, the UAV gets into the landing window and starts preparing for the landing approach, which means the lateral error should be further eliminated. Here, a fuzzy controller is designed and the parameters of fuzzy controllers are set based on experience. The following figures shows the Membership functions of the fuzzy controller. The lateral error, differential and integral quantity of the lateral error are three inputs of the fuzzy controller, and the deflection of the ailerons is the output.
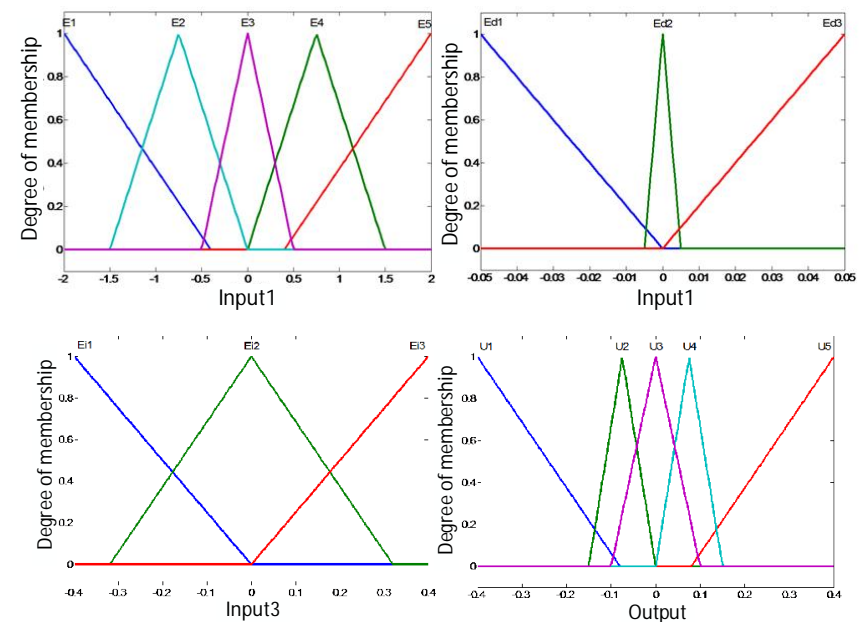

FIGURE XVIII. MEMBERSHIP FUNCTION OF THE FUZZY CONTROLLER

Table 11 and Table 12 shows the rules and other parameters of the fuzzy logic control of Fuzzy controller.

TABLE XI. PARAMETERS AND RULES OF THE FUZZY CONTROLLER

\begin{tabular}{|c|c|c|c|c|c|c|c|}
\hline Input1 & Input2 & Input3 & Output & Input1 & Input2 & Input3 & Output \\
\hline E1 & Ed1 & Ei1 & U1 & E3 & Ed 2 & Ei3 & U4 \\
\hline E1 & Ed1 & Ei2 & U1 & E3 & Ed 3 & Ei1 & U4 \\
\hline E1 & Ed1 & Ei3 & U1 & E3 & Ed 3 & Ei2 & U4 \\
\hline E1 & Ed2 & Ei1 & U1 & E3 & Ed 3 & Ei3 & U4 \\
\hline E1 & Ed2 & Ei2 & U1 & E4 & Ed 1 & Ei1 & U3 \\
\hline E1 & Ed2 & Ei3 & U2 & E4 & Ed 1 & Ei2 & U3 \\
\hline E1 & Ed3 & Ei1 & U3 & E4 & Ed 1 & Ei3 & U3 \\
\hline E1 & Ed3 & Ei2 & U3 & E4 & Ed 2 & Ei1 & U4 \\
\hline E1 & Ed3 & Ei3 & U3 & E4 & Ed 2 & Ei2 & U4 \\
\hline E2 & Ed1 & Ei1 & U1 & E4 & Ed 2 & Ei3 & U5 \\
\hline E2 & Ed1 & Ei2 & U1 & E4 & Ed 3 & Ei1 & U5 \\
\hline E2 & Ed1 & Ei3 & U1 & E3 & Ed2 & Ei2 & U3 \\
\hline E2 & Ed2 & Ei1 & U2 & E3 & Ed2 & Ei3 & U4 \\
\hline E2 & Ed2 & Ei2 & U2 & E3 & Ed 3 & Ei1 & U4 \\
\hline E2 & Ed2 & Ei3 & U2 & E3 & Ed3 & Ei2 & U4 \\
\hline E2 & Ed3 & Ei1 & U2 & E3 & Ed3 & Ei3 & U4 \\
\hline E2 & Ed3 & Ei2 & U2 & E4 & Ed 1 & Ei1 & U3 \\
\hline E2 & Ed3 & Ei3 & U3 & E4 & Ed 1 & Ei2 & U3 \\
\hline E3 & Ed1 & Ei1 & U2 & E4 & Ed 1 & Ei3 & U3 \\
\hline E3 & Ed1 & Ei2 & U2 & E4 & Ed2 & Ei1 & U4 \\
\hline E3 & Ed1 & Ei3 & U2 & E4 & Ed2 & Ei2 & U4 \\
\hline E3 & Ed2 & Ei1 & U3 & E4 & Ed2 & Ei3 & U5 \\
\hline E3 & Ed2 & Ei2 & U3 & & & & \\
\hline
\end{tabular}


TABLE XII. PARAMETERS OF FUZZY CONTROLLER IN HEADING CHANNEL

\begin{tabular}{|c|c|c|c|c|}
\hline $\begin{array}{c}\text { And } \\
\text { method }\end{array}$ & $\begin{array}{c}\text { Or } \\
\text { method }\end{array}$ & Implication & Aggregation & Defuzzification \\
\hline $\min$ & $\max$ & $\min$ & $\max$ & centroid \\
\hline
\end{tabular}

\section{Simulation Results}

The simulation results show that the method proposed can guide the UAV to approach the carrier with energy-saving and smooth path, and at the final stage, the lateral error is small enough to prepare for the landing approach.

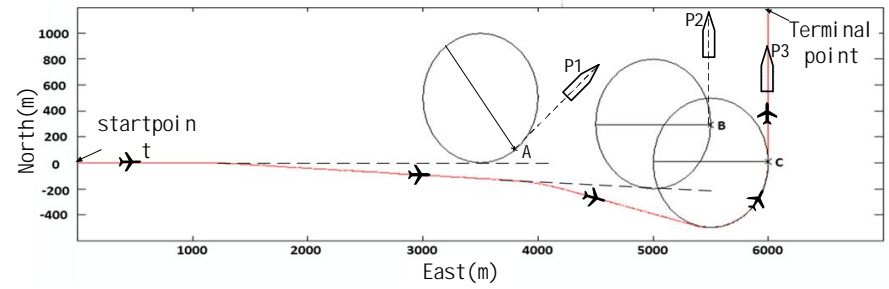

FIGURE XIX. THE SIMULATION RESULTS OF THE TRAJECTORY OF THE UAV

In the simulation, because of the change of the velocity of the carrier and the UAV, the estimated landing window moves from $A$ to $B$ and finally settle at the point $C$ and the red line shows the track of the UAV. Figure above shows the UAV can adjust its direction according to the change of carrier velocity, and approach the carrier in a short and smooth path.

The response of each channel is shown in the figures below:
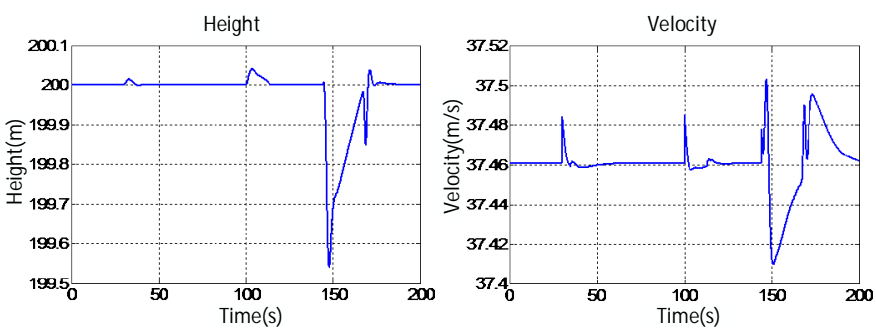

\section{FIGURE XX. SIMULATION RESULTS OF THE HEIGHT AND} VELOCITY CHANNEL

Figures above illustrate the response of the height and velocity of the UAV during the flight. The height maintains near $200 \mathrm{~m}$ with errors not more than $0.5 \mathrm{~m}$ and the velocity keeps near $37.46 \mathrm{~m} / \mathrm{s}$.
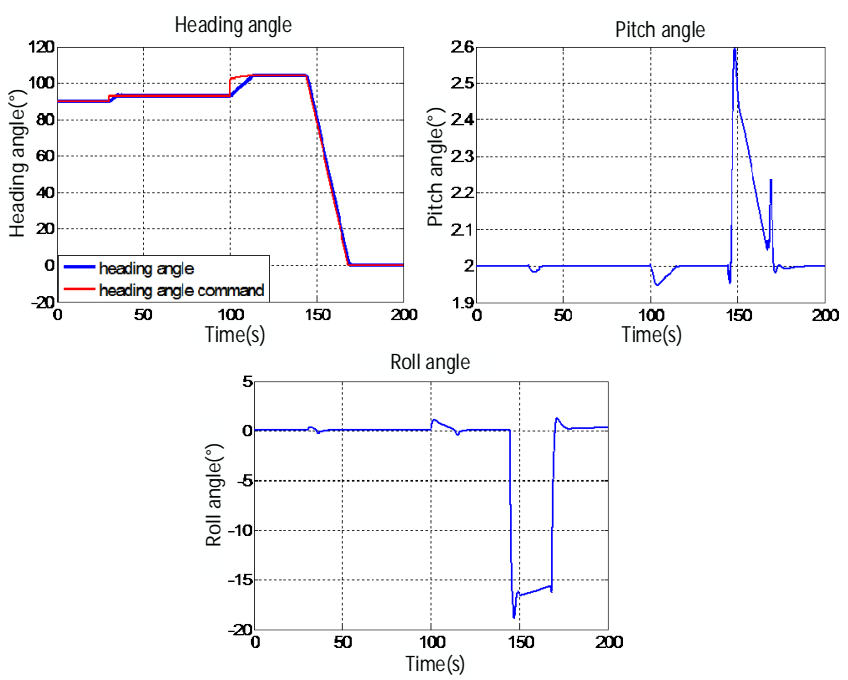

FIGURE XXI. SIMULATION RESULTS OF THE THREE ATTITUDE CHANNELS

Figures above illustrate the response of the three attitude channels, which show that the UAV flies in the safe and stable state.

In the last stage, the lateral errors are further eliminated by the fuzzy controller, and the following figures show the effects of the controller.
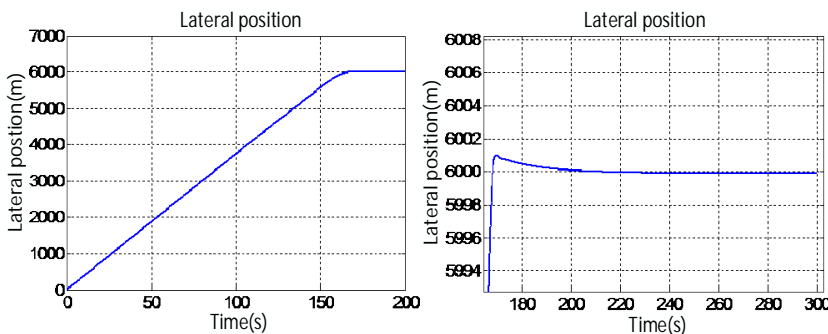

FIGURE XXII. EFFECTS OF THE FUZZY CONTROLLER ON LATERAL ERROR ESTIMATION

The UAV gets into the landing window at $168 \mathrm{~s}$, and $12 \mathrm{~s}$ after that, the lateral error converges to $0.5 \mathrm{~m}$ which is enough for the preparation of the further landing approach. And 12s after the UAV getting into the landing window, the distance between the UAV is $450 \mathrm{~m}$ and there are about $550 \mathrm{~m}$ away from the carrier, in which section the UAV can start decelerating and preparing for the landing.

\section{CONCLUSION}

In this paper, an innovative path planning and control method which ensure UAV to approach the carrier and achieve accompanying flight before landing is proposed. Firstly, the landing window is estimated according to the position and velocity of them and updated if the velocity changes. Secondly, the UAV is guided to fly along the planned path towards the estimated landing window. Here we propose a path planning method which can ensure the UAV approach the carrier in an energy-saving and smooth way. To keep the UAV fly along the planned path, a control strategy is proposed based on three 
stages in the path, which are tangent flight, circular flight, and accompanying flight. To further eliminate the lateral error in the accompanying flight, a fuzzy controller is used to deal with the non-linearities in the UAV system.

The simulation results show that the path planning and control method proposed can guide the UAV to approach the carrier and achieve accompanying flight successfully and smoothly. In the final stage, after the UAV accompany the carrier for $12 \mathrm{~s}$, the lateral error can be reduced to $0.5 \mathrm{~m}$, which is small enough for the landing. And throughout the whole process of the flight, the attitude of the UAV is stable without large maneuver.

\section{REFERENCES}

[1] Eric W. Frew, Dale A. Lawrence, Steve Morris, "Coordinated standoff tracking of moving targets using Lyapunov guidance vector fields," Journal of guidance, control, and dynamics, 31(2), 290-310 (2008).

[2] Xiaowei Fu, Huicheng Feng, Xiaoguang Gao, "UAV Mobile Ground Target Pursuit Algorithm,” J Intell Robot Syst, 68, 359-371 (2012).

[3] BENJAMIN D. REINEMAN, LUC LENAIN, W. KENDALL MELVILLE, "The Use of Ship-Launched Fixed-Wing UAVs for Measuring the Marine Atmospheric Boundary Layer and Ocean Surface Processes," American Meteorological Society, 2029-2049(2016).

[4] Jason Wadley, Greg Tallant, Robert Ruszkowski, “ Adaptive flight control of a carrier-based unmanned air," AIAA Guidance, Navigation, and Control Conference and Exhibit, 2003, 10.2514/6.2003-5596.

[5] Jovan Boskovic, Joshua Redding, “An Autonomous Carrier Landing System for Unmannned Aerial Vehicles," AIAA Guidance, Navigation, and Control Conference, 2009, 10.2514/6.2009-6264.

[6] Tri D. Ngo, Cornel Sultan, "Nonlinear Helicopter and Ship Models for Predictive Control of Ship Landing Operations," AIAA Guidance, Navigation, and Control Conference, 2014, 10.2514/6.2014-1298.

[7] Dong Jia, Juris Vagners, "Parallel Evolutionary Algorithms for UAV Path Planning," AIAA 1st Intelligent Systems Technical Conference, 2004, 10.2514/6.2004-6230.

[8] Joseph Stern, Kelly Cohen, "FLC-based Landing Approach and Collision Avoidance Path Planner for Multiple Aircraft and Runways," 50th AIAA Aerospace Sciences Meeting including the New Horizons Forum and Aerospace Exposition, 2012, 10.2514/6.2012-489.

[9] George Hellstern, Ron Wilson, Frederick Harris, "Adaptive Motion Planning Approaches for Small UAV Flight," Infotech@Aerospace 2012, 2012, 10.2514/6.2012-2477 